\title{
Optimization of Unicondylar Knee Replacement according to Con- tact Pressure
}

\author{
Michal Ackermann ${ }^{1 \mathrm{~A}}$, Lukáš Čapek ${ }^{1}$ \\ ${ }^{1}$ Technical University of Liberec, Dept. of applied mechanics, Studentska 2, 46117 Liberec 1, Czech Republic
}

Received 15 September 2010, Accepted 20 November 2010

\begin{abstract}
The project deals with contact pressure analysis on two constructional types of modern type of knee prosthesis - the unicompartmental knee replacement. In the first part of the project the contact pressure distribution on replacements is analyzed in two ways. The first method is experimental with using pressure sensitive Pressurex ${ }^{\circledR}$ films. Second approach is a numerical analysis with the support of finite element method (FEM) made in ANSYS Multiphysics 10.0 software package. Results from both approaches are compared at the end of the first part. In the second part the chosen type of replacements is optimized according to contact pressure. The goal was to reduce the contact pressure and to improve its distribution for taking its full advantages.
\end{abstract}

Key words: Biomechanics, Knee joint, Unicompartmental replacement, FEM, Contact pressure

\section{Introduction}

Nowadays we're able to increase the quality of life for those people who are disabled with knee joint failure thanks to research of artificial joints. These surgical interventions, which are replacing damaged joint surfaces of femoral, tibial bone and related soft tissues, belongs among today's standard orthopedic operations. The most common reasons for implantation of knee joint replacement are:

- $\quad$ Degenerative knee diseases (e.g. osteoarthritis);

- Destruction of the joint by the reason of rheumatic disease;

- $\quad$ Damage of the joint caused by an accident;

- And others.

Present experiences in the field of orthopedics and biomechanics lead to the progression of so-called mini-invasive operations. This means, that on specific cases, there's a possibility to use partial (unicondylar) knee replacements instead of total knee replacement. Quick process of operation, short term of convalescence and of course lower price of the implant are main reasons for further research of these modern replacements.

An unicondylar replacement generally consists of three parts: femoral component, tibial platform and between them inserted part made of an ultra-high molecular weight polyethylene (UHMWPE).

In this work, two constructional types of partial knee replacement were tested (Figure 1). These types differ by a movement ability of UHMWPE insert on tibial platform. The first of the two types is characterized by a possibility of free horizontal movement of insert on tibial platform, while in the second case the insert is fully fixed. Very important aspect is a shape of PE insert's contact area. If it's designed in a wrong way it could lead to fast failure. This is of course unacceptable so the question is which one of the mentioned constructional solutions is more suitable for use.
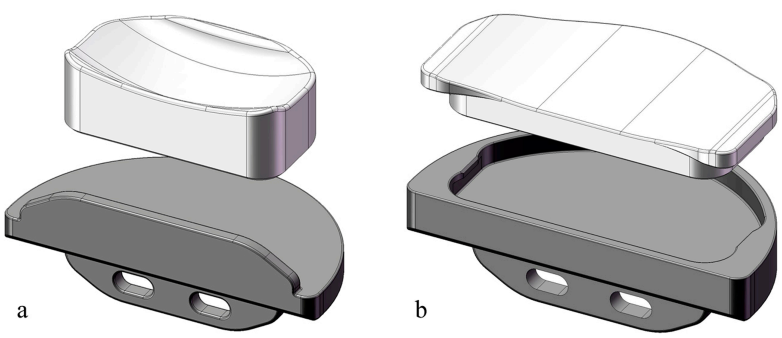

Fig.1. Two constructional options of partial knee replacement

Many works dealing with knee replacements indicate the value of contact pressure as one of the main signs of premature wear of PE insert (Lin, Y. [4], Shi, J. [7]). The aim of this article is to analyze distribution of contact pressure on tested replacements and to consider which of these two types is more suitable for use. Two methods were used: the experimental method, where contact pressure is measured by Pressurex films and by a numerical approach - finite element method (FEM).

\subsection{Pressurex films}

These films which react on load between contacting bodies are offering an easy way to find out contact pressure distribution and its value. Film itself is made of a material called Mylar (firm and highly durable polyester). It contains a layer of microcapsules filled with pigment. When a force is applied these microcapsules tear and pigment creates instantaneous and permanent topographical image of contact pressure distribution in contact area of bodies (Fig. 2).
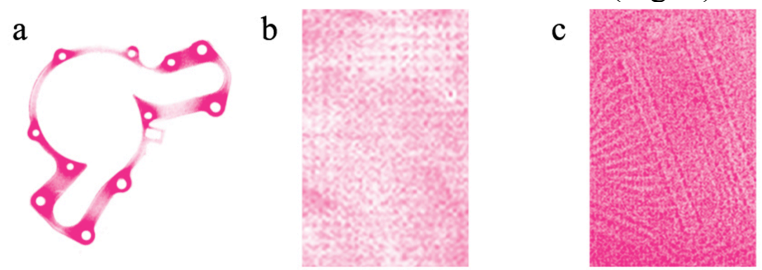

Fig.2. Examples of Pressurex film applications

\footnotetext{
${ }^{\mathrm{a}}$ Corresponding author: michal.ackermann@gmail.com
} 


\subsection{Finite element method (FEM)}

Solving the problem with defined contact belongs in FEM, considering nonlinearities which it brings, among one of its most computational and time consuming tasks. The contact definition is necessary in those cases, where it's impossible to replace it with some of the boundary conditions, for example:

- Dynamic impact simulation,

- Contact of bodies with defined coefficient of friction,

- Interaction of assembly components,

- $\quad$ Etc.

\section{Materials and methods}

Experiments and numerical analyzes were done in the same way:

- Replacements were tested in five positions of knee joint flexion;

- Parts of the replacement was pushed towards each other by a force $1270 \mathrm{~N}$ which is equal to those in joint in full extension while standing;

- On a type with free PE insert there's difficult to choose an area of contact between PE housing and femoral component. By the reason of large time consumption when testing all potential contact points, only one of them was chosen.

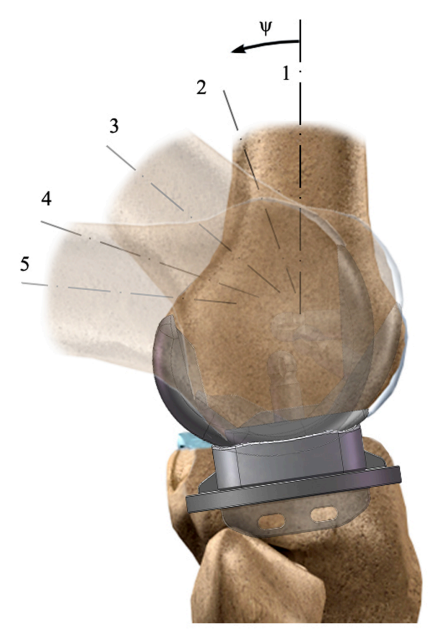

Fig.3. Five testing positions shown on knee joint

Table 1. Degrees of flexion

\begin{tabular}{cc}
\hline Position number & $\begin{array}{c}\text { Flexion angle } \\
\psi[\mathrm{deg}]\end{array}$ \\
\hline 1 & 0 \\
2 & 20 \\
3 & 50 \\
4 & 70 \\
5 & 85 \\
\hline
\end{tabular}

\subsection{Experiments}

Experiments were performed on uniaxial testing machine (TIRA test, Germany) on the Department of Applied Mechanics, Technical University of Liberec, Czech Republic. A device allowing variable positioning of the femoral component against tibial plate and PE insert simulating knee flexion/extension was built (Figure 3). The Pressurex ${ }^{\circledR}$ indicating film, cut into necessary size, was inserted between PE housing and femoral component and then pushed against each other to contact under the load of $1270 \mathrm{~N}$. The load was applied for over ten seconds so the topographical image of contact surface was developed on film. This process was repeated for five angles of flexion (Fig. 3). All obtained films were analyzed and distribution of contact pressure was reached.

In the case of partial knee replacement with free PE housing a LOW type $(2,5-10 \mathrm{MPa})$ of Pressurex ${ }^{\circledR}$ film was used By the reason of the presumption of higher contact pressures, MEDIUM type $(10-50 \mathrm{MPa})$ was used for type with fixed PE insert.
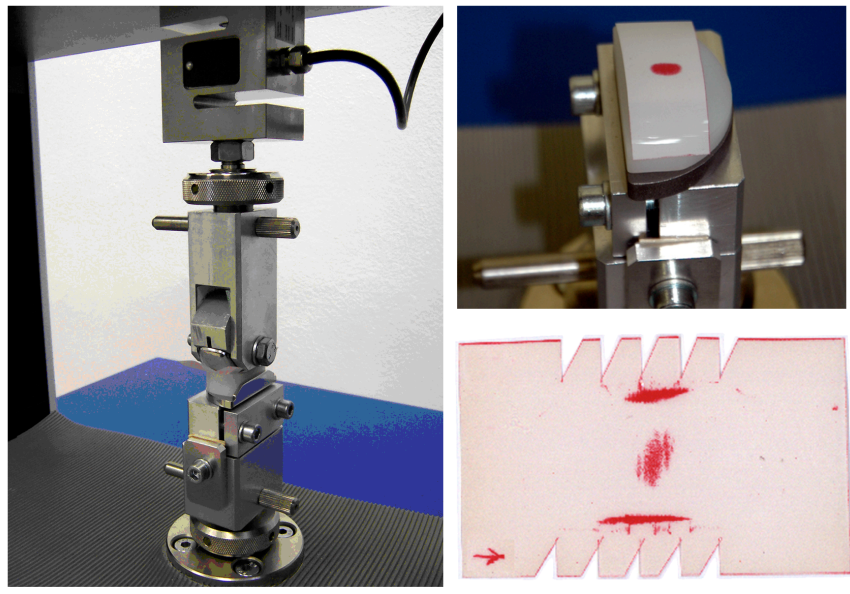

Fig.4. Snapshots of experiments

\subsection{FEM analysis}

After the experiments, FEM analyzes were proceeded too. The task was solved in ANSYS 10.0 software package. First of all, assemblies of the parts of tested replacements were crated in CAD/CAM software Pro/ENGINEER. Each assembly corresponded to a concrete angle of flexion. These entities were then exported directly into ANSYS software. Following premises were established:

- Material of the parts was considered as elastic and isotropic;

- In the case of fixed PE insert the contact of insert and tibial platform is replaced by boundary conditions, which fixes its movement in a more simple way;

The force $1270 \mathrm{~N}$ was converted to pressure load and applied on top surfaces of femoral component.

Models were meshed by 10-noded tetrahedron shaped volume elements. Resultant mesh was locally refined in the area of contact to gain more accurate results of contact pressure. 
Material models are characterized by constants, summarized in table 2.

Table 2. Material properties of tested components

\begin{tabular}{lcl}
\hline \multicolumn{3}{c}{ Tibial plate and femoral component } \\
(Co-Cr-Mo alloy) \\
\hline Young's modulus & E [MPa] & 228000 \\
Poisson's ratio & $\mu[-]$ & 0,3 \\
\hline \multicolumn{3}{c}{ PE housing } \\
\multicolumn{3}{c}{ (ultra-high molecular weight polyethylene - UHMWPE) } \\
\hline Young's modulus & E [MPa] & 500 \\
Poisson's ratio & $\mu[-]$ & 0,3 \\
\hline
\end{tabular}

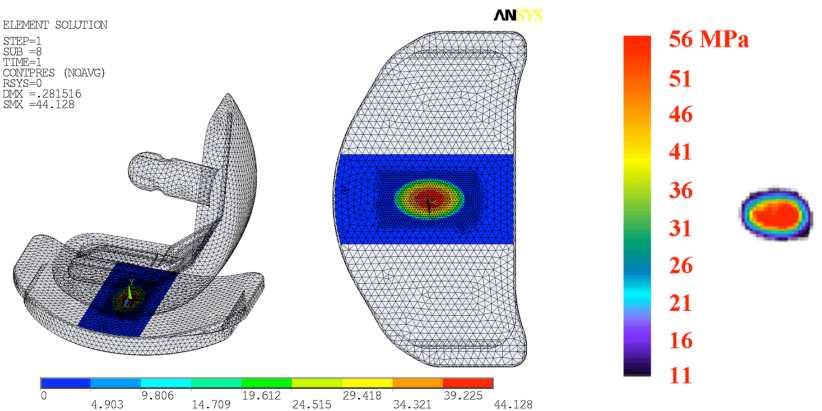

Fig.7. Fixed PE housing; Position 5

In each defined contact, coefficient of friction $\mathrm{f}=0.05$ was used. This is essential in case of the type with free PE housing. It was impossible to set up the PE insert into proper position against femoral component in Pro/ENGINEER software. But thanks to defined friction there's a possibility for insert to move during the task evaluation and therefore gain the right position.
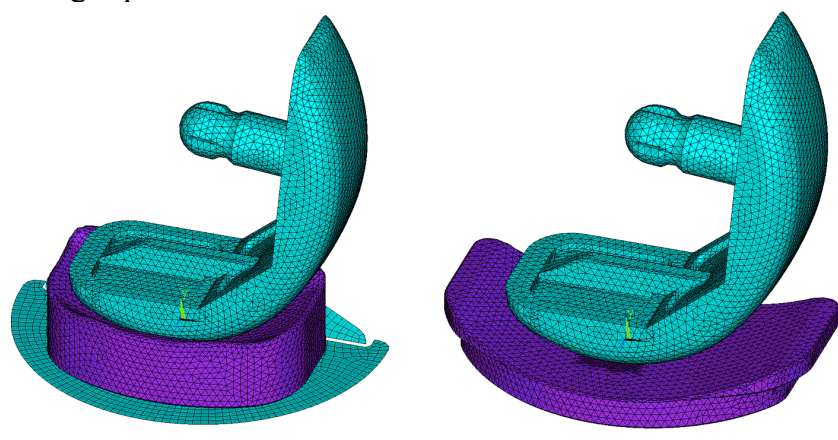

Fig.5. Meshed models of the both unicondylar knee replacement types

\section{Results}

The first set of results (Figure 6,7) belongs to the partial knee replacement with fixed PE insert. On the left side can be seen result from FEM analysis, right side shows contact pressure distribution gained thanks to Pressurex films. Second set (Figure 7,8) shows results of the free PE insert type.

The maximal values of contact pressure goes up to $36 \mathrm{MPa}$ in case of free housing and $56 \mathrm{MPa}$ in case of fixed housing. In case of free housing, we can see that results of contact pressure distribution are not, especially in position 5 , in a good agreement. In case of fixed housing, results are more acceptable.

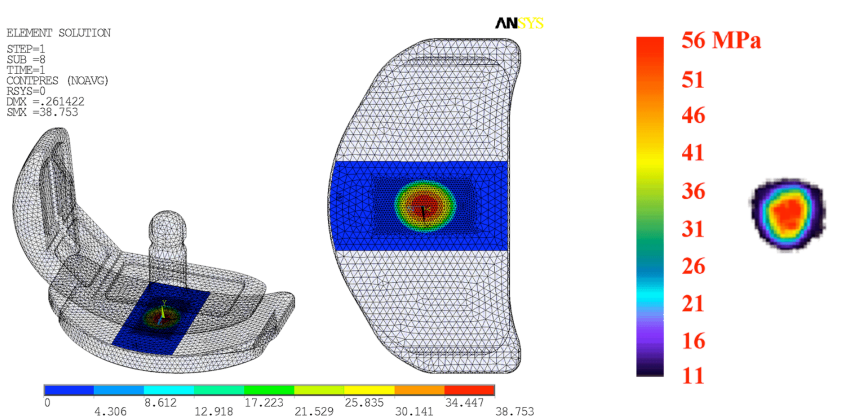

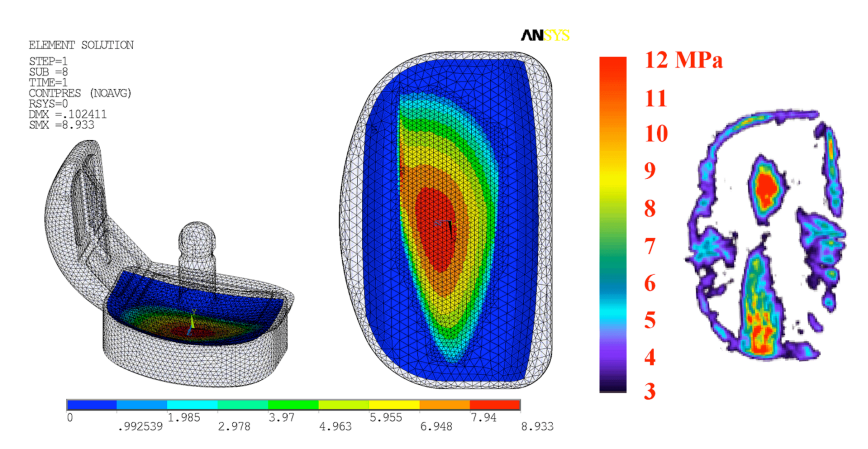

Fig.8. Free PE housing; Position 1

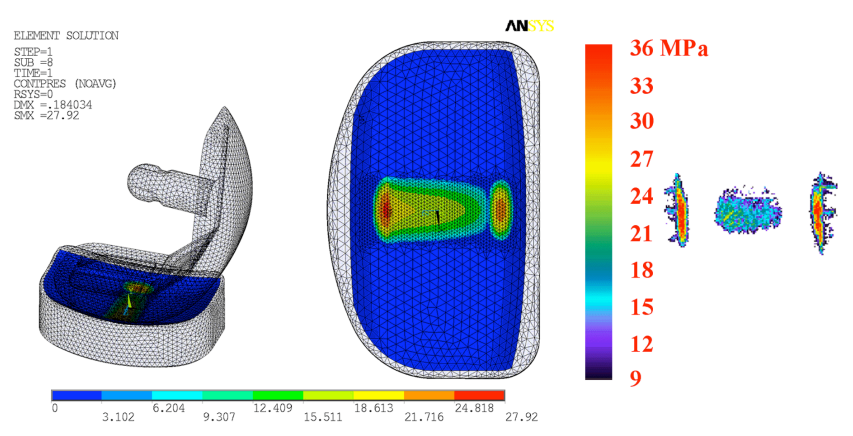

Fig.9. Free PE housing; Position 5

\section{Optimization}

According to previous results, the uni-compartmental knee replacement with free PE housing was chosen for the optimization process. Shape of contact surface, which follows convex shape of femoral component, guarantees more advantageous distribution of contact pressure and thereby reduction of its value. The shape of PE insert was modified only. Contact surface of the femoral part follows the shape of healthy condyle surface. Its modifications should therefore lead to changes in joint kinematical properties which isn't suitable.

Fig.6. Fixed PE housing; Position 1 


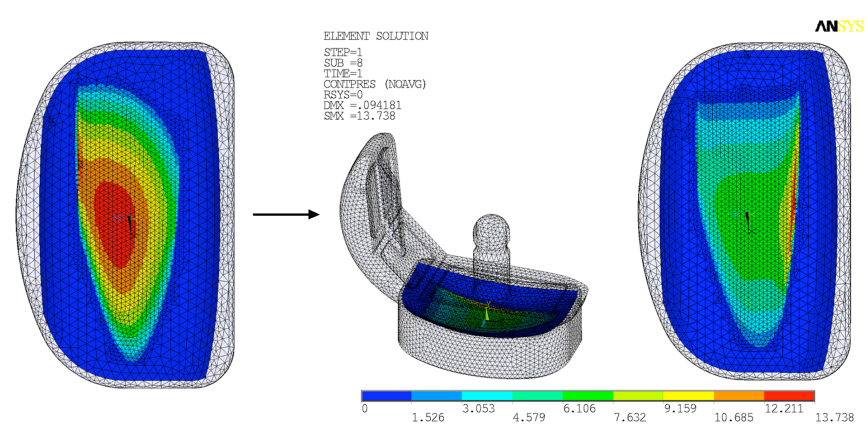

Fig.10 Result of optimization on a type with free PE insert in position 1
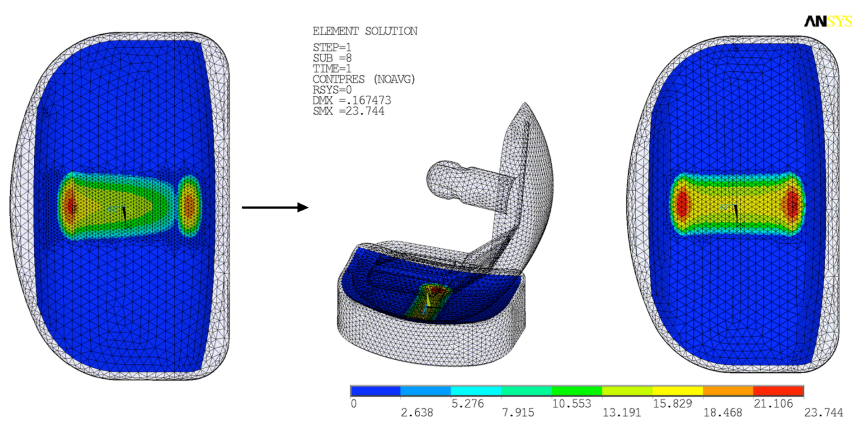

Fig.11 Result of optimization on a type with free PE insert in position 5

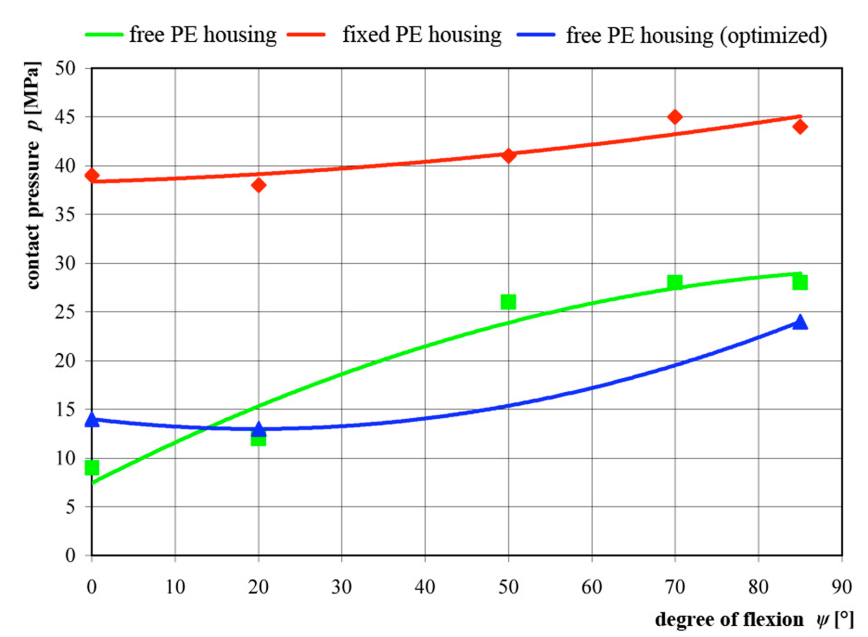

Fig.12 Results of contact pressure gained from FEM analyzes

\section{Conclusions}

An unicondylar knee replacement substitute only a half of the knee joint. This type of replacement is used when the 7 damage is limited only to one side of the joint. This allows maintaining the healthy part of the knee, shortening the time and pricing. However, even with only half of the joint de- 8 . stroyed, many surgeons prefer doing a total knee replacement believing this is a better procedure than the half-knee (unicondylar) replacement. Nevertheless there are surgeons who believe it is more appropriate to perform a unicondylar knee replacement in the right circumstance.
In the article a contact pressure distribution on both tested types of partial knee replacement was performed. An experimental and a numerical approach were used for evaluation of contact pressures.

In general, performed experiments offered, comparing to FEM analyses, higher values of contact pressure. The highest difference between the two approaches was $18 \mathrm{MPa}(32 \%)$. The type with a free housing seems to be more suitable for use thanks to its more effective contact pressure distribution. Graphical presentation of results gained from FEM analyzes can be seen on figure 12. Comparing of results with works of other authors (Guo, Y. et al. [1], Hopkins, A. R. et al. [2]) didn't show any major differences of values of contact pressures.

One of the main advantages of this work is that a new experimental approach was used compare to the usual numerical approach. FEM analysis is of course great apparatus but its results could be distorted by many factors. The most critical is the selection of boundary conditions, definition of contacts and finding a material model.

Testing of these modern knee replacements by static load in order to reveal the distribution of contact pressure is of course only one of many views to judge their usability. It's then a question how the chosen replacement should stand in other aspects (e.g. dynamic analyses, interaction with skeleton system, influence to biomechanics of joint, etc.). These are some of the subjects for our future work in the field of partial knee replacements.

\section{References}

1. Galvin A. L., Kang L., Effect of conformity and contact stress on wear in fixed-bearing total knee prostheses, Journal of Biomechanics, 42(12), 1898-1902, (2009).

2. Guo Y., Zhang X., Chen W.: Three-Dimensional Finite Element Simulation of Total Knee Joint in Gait Cycle, Acta Mechanica Solida Sinica, 22(4), 347-351, (2009).

3. Hopkins, Andrew R., New, Andrew M., Rodriguez-yBaena F., Taylor M.: Finite element analysis of unicompartmental knee arthroplasty, Medical Engineering \& Physics, 32(1), 14-21, (2010).

4. Lin, Y.: Experimental evaluation of a natural knee contact model using response surface optimization, $\mathrm{PhD}$ thesis, University of Florida (2004)

5. Miyoshi, S., Takahashi, T., Ohtani M., Yamamoto H., Kameyama K.: Analysis of the shape of the tibial tray in total knee arthroplasty using a three dimension finite element model, Clinical Biomechanics, 17(7), 521-525, (2002).

6. Rakotomanana, R.L., Leyvraz, P.F., Curnier, A., Heegaard, J.H., Rubin, P.J.: A finite element model for evaluation of tibial prosthesis-bone interface in total knee replacement, Journal of Biomechanics, 25(12), 1413-1424, (1992).

Shi J.: FEM of total knee replacement considering gait cycle load and malalignment, $\mathrm{PhD}$ thesis, University of Wolverhampton, (2007)

. Simpson, D.J., Gray, H., D'Lima, D., Murray D.W., Gill, H.S.: The effect of bearing congruency, thickness and alignment on the stresses in unicompartmental knee replacements, Clinical Biomechanics, 23(9), 1148-1157, (2008). 